\title{
MATHEMATICAL MODELLING OF GLIOBLASTOMA TUMOUR DEVELOPMENT: A REVIEW
}

\author{
HARALAMPOS HATZIKIROU* ${ }^{*}$ and ANDREAS DEUTSCH ${ }^{\dagger}$ \\ Center of Information Services and High Performance Computing (ZHI), \\ Technical University-Dresden, Zellescher Weg 12, 01069, Dresden, Germany \\ *haralampos.hatzikirou@tu-dresden.de \\ $\dagger^{\dagger}$ andreas.deutsch@tu-dresden.de \\ CARLO SCHALLER ${ }^{\ddagger}$ and MATTHIAS SIMON $\S$ \\ Department of Neurosurgery, University of Bonn Medical Center, \\ Sigmund-Freud-Str. 25, 53105, Bonn, Germany \\ ¥carlo.schaller@ukb.uni-bonn.de \\ §matthias.simon@ukb.uni-bonn.de \\ KRISTIN SWANSON \\ Departments of Pathology and Applied Mathematics, \\ University of Washington and Laboratory of Neuropathology, \\ Harborview Medical Center, Seattle, WA 98104-2499, USA \\ swanson@amath.washington.edu \\ Received 5 April 2005 \\ Communicated by N. Bellomo and P. Maini
}

\begin{abstract}
During the past several years mathematical models have been applied to various aspects of cancer dynamics, in particular avascular and vascular tumour growth, invasion, angiogenesis, and metastasis. This paper focuses on the most common and malignant brain tumour, glioblastoma, and surveys the growing number of studies dealing with mathematical modelling of this tumour. We attempt to classify these studies by their biomedical relevance and critically analyse their results. The aim of this review is to provide a meaningful reference, to both biomedical and mathematical researchers, of the current state of the art of glioma tumour modelling. The discussion attempts to identify current open problems as well as new research perspectives in the mathematical modelling of glioblastoma growth.
\end{abstract}

Keywords: Glioblastoma multiforme; mathematical modelling; avascular tumour growth; invasion; therapy.

AMS Subject Classification: 92C50

\section{Introduction}

More than 120 different tumour types can be found in the human brain. Gliomas are generally considered to originate from glial cells or their precursors and account for 
$30-40 \%^{48,69}$ of all intracranial neoplasms. Currently, much research is devoted to a better understanding of the molecular mechanisms leading to the formation, further growth and malignant transformation of brain tumours. Of particular interest are the genes and genetic alterations involved in this process. In addition to clinical observations, experimental methods have been developed to allow the investigation of tumour invasion, including cell cultures of multicellular spheroids for avascular tumours, endothelial cells for angiogenesis ${ }^{3}$ and other culture methods as described in Medico et al. ${ }^{35}$ However, attempts to correlate these in vitro data with clinical observations have yielded unsatisfactory results.

A better understanding of the dynamics of tumour formation and growth can be expected from a mathematical (i.e. quantitative) modelling. Mathematical models could ultimately improve the overall clinical outcome by predicting the results of specific forms of treatment administered at specific time points. During the last decade theoreticians have developed a great variety of tumour models covering various morphological and functional aspects of tumour growth. These advances have been recently reviewed ${ }^{37,47}$ with a focus on the classification of mathematical tools and computational algorithms.

The most common and also the most malignant glioma is the Glioblastoma multiforme (GBM), which is the clinical focus of this review. Thus we have become previously interested in the modelling ${ }^{59,68}$ of this tumour. We will review the different GBM models, classify them by their biomedical relevance, and discuss the model results. The structure of this paper is as follows: we start with a description of various relevant clinical and biological aspects of GBMs, followed by a short review of general tumour growth models. Then a critical analysis of existing GBM models is provided. Finally, in the discussion, we present some ideas concerning the future of GBM mathematical modelling.

\section{Glioblastoma Multiforme: A Clinical and Biological Description}

\subsection{Growth pattern}

The World Health Organisation (WHO) distinguishes four grades of malignancy: WHO grade I-IV. ${ }^{23}$ Glioblastoma multiforme (GBM) WHO grade IV is the most frequent glioma and accounts for more than $50 \%$ of all primary brain tumours. Many WHO grade II or III gliomas finally become GBMs through a process termed malignant progression.

GBMs grow in three-dimensional, irregular patterns and infiltrate the surrounding brain tissue. ${ }^{20,51,54}$ The tumours frequently seem to grow along the fibres of the white matter ${ }^{18,34,50}$ or along vessels, i.e. appear to follow physical structures in the extracellular matrix of the neighbouring brain. ${ }^{36}$ During the evolution of the disease, putatively multifocal growth is sometimes observed; however, in most instances these solid tumour nodules are not separated by healthy brain but rather by diffusively infiltrated CNS (Central Neural System) tissue which gives the false impression of multifocality. 


\subsection{Glioblastoma: Therapy}

Standard therapy for GBMs is microsurgical resection followed by conventional external beam radiation. Chemotherapy is often prescribed for recurrent tumour, and has also been used with some success in an adjuvant setting. ${ }^{56}$ Median overall survival does not exceed 10-16 months although a percentage of patients may survive for several years. ${ }^{38,56,65}$ Time to tumour recurrence has been correlated with the extent of the resection in retrospective studies. ${ }^{25}$

The proximity of eloquent areas and structures of the brain limit the neurosurgeon's ability to resect GBMs. Patients with a limited life expectancy frequently will not tolerate a significant reduction of their quality of life related to a serious neurological deficit. Surgical strategies have been traditionally chosen based on personal or institutional experience (i.e. fairly subjective parameters) rather than a formalised analysis of the aforementioned factors. The effects of radiation and chemotherapy also depend on tumour location, size, geometry and tissue structure, as well as biological factors. The radiation dose that can be delivered safely (and there is a dose-effect relationship for GBM radiotherapy) is ultimately limited by the radiosensitivity of neighbouring tissues.

\section{Mathematical Modelling: Tumour Growth}

Cancer research offers fertile ground for various mathematical modelling approaches. The complexity of the tumour evolution process involves many different phenomena, which occur at different spatio-temporal scales including processes at the sub-cellular, cellular and tissue levels. Mathematical models should allow description and distinction of these levels. Accordingly, one can distinguish: microscopic, mesoscopic and macroscopic scales respectively. ${ }^{47}$ In particular:

- The microscopic scale refers to phenomena that occur at the sub-cellular level and to the activities that take place within the cell. The microscopic scale concentrates on molecular interactions and resulting phenomena, such as alterations of signalling cascades and cell cycle control, gene mutations, etc.

- The mesoscopic scale refers to cellular interactions and therefore to the main dynamics of cell populations, e.g. adhesion, contact inhibition, chemotaxis etc.

- The macroscopic scale focuses on tissue level processes taking into account macroscopic quantities, such as volumes, flows etc. Continuum phenomena include cell convection and diffusion of nutrient and chemical factors, mechanical stress and the diffusion of metastases.

Cancer dynamics involve phenomena at each of these scales and a coupling of the above-mentioned scales. For example, genetic alterations may lead to mutated cells (microscopic scale), aggregation of these cells (mesoscopic scale) and initiation of tumour formation. Models that deal with phenomena at multiple scales are called multi-scaled.

The goal of mathematical modelling and simulation is to offer a better understanding of tumour growth which will ultimately improve the therapeutic outcome. 
Aspects of cancer that have attracted the interest of researchers include: genetic alterations and their effects, early avascular tumour growth, proliferation, invasion, angiogenesis, vascular growth and metastasis. The literature also contains several attempts to model therapies for specific kinds of cancer, e.g. GBM. In this review, we attempt to classify the existing models by their focus on specific biological or clinical aspects.

There are many mathematical modelling studies that deal with sub-cellular processes, such as gene mutations, cell cycle disturbances etc. (e.g. Alarcon et al. ${ }^{2}$ ). Some studies consider genetic instabilities of tumour (accumulation of genetic alterations) through the introduction of cell subpopulations and analyse the resulting spatio-temporal patterns. ${ }^{4,44}$

Recently, many researchers have studied and modelled the early growth of avascular tumour. At that stage, the progress of tumour growth depends on the history of each individual cell and its local interactions with environmental variables as well as with neighbouring tumour cells. Corresponding cell-based models have been used to successfully describe early tumour growth by Kansal et al., ${ }^{22}$ Sander et al. ${ }^{49}$ Dormann et al. ${ }^{14}$ Typically, these models consider a small set of mutated cells (primary tumour) and analyse the growth patterns, the goal being to explain the growth pattern of multicellular tumour spheroids as seen experimentally in vitro and attempt to simulate in vivo tumour evolution.

There are many mathematical models that describe the temporal or spatiotemporal dynamics of tumour proliferation and invasion. Much of the experimental data that exists has been modelled using purely time-dependent growth laws based on the assumption of either exponential or Gompertzian growth. ${ }^{33}$ Additionally, the spatio-temporal evolution of proliferation has been modelled as a behaviour that emerges from local micro-interactions. ${ }^{22}$ Deterministic reaction-diffusion models have been used to model the spatial growth of tumours usually assuming that tumour growth is a wave propagation phenomenon ${ }^{31,45,46,52,53}$ and is driven by a random movement of malignant cells. ${ }^{63}$ Whilst these models are able to capture the tumour structure at the tissue level, they fail to describe the tumour at the cellular and the sub-cellular levels. Cellular automata models can provide such a description and allow a more realistic stochastic approach at the cellular level. ${ }^{43,55}$ Recently, Swanson et al. ${ }^{58}$ modelled proliferation and migration of tumours in a 3D diffusion framework, within an inhomogeneous environment. The works of Turner et al., ${ }^{64}$ Mansuri et al. ${ }^{20-30}$ focus on tumour proliferation and invasion and treat them at a cellular and sub-cellular level.

Several mathematical models have been developed to describe tumour-induced angiogenesis and vasculogenesis. Many of these models use a continuum, deterministic framework in one space dimension. ${ }^{10,40}$ Although these models are capable of capturing some features of angiogenesis such as average sprout density and network expansion rates, they are unable to provide more detailed information concerning the actual structure and morphology of the capillary network and as such are of limited predictive value. More realistic continuum models of angiogenesis in two space 
dimensions have been described by Orme \& Chaplain. ${ }^{41}$ The results of these models may permit a more detailed qualitative comparison with in vivo observations concerning the spatio-temporal distribution of capillary sprouts within a network. A mechanochemical model of angiogenesis and vasculogenesis has been recently developed by Manoussaki. ${ }^{27}$ However, even the latest models, do not adequately treat certain important events, such as repeated sprout branching and hence the overall dendritic structure of the vascular network. The advent of cellular automata models of angiogenesis delivered realistic simulations and the treatment of the problem has become multi-scaled. ${ }^{2}$ The morphogenetic mechanism of angiogenesis has been studied through in vitro experiments of endothelial cells cultured on basement membrane proteins. An excellent study of Ambrosi et al. ${ }^{3}$ provides a model of such experiments. The work of Painter et al. ${ }^{44}$ provides development scenarios of gene mutations that lead towards a vascularized tumour.

Metastasis is a complex process that involves many factors: detachment, migration, intravasation, transport and arrest, extravasation, invasion of the target organ. ${ }^{47}$ In the work of Anderson, ${ }^{4}$ the author has focused on the role of cell adhesion during the formation of metastases. This model simulates how the reduction of adhesion strength will ultimately lead to the detachment of tumour-cell clusters.

Recently, modelling cancer therapies and specifically the treatment of brain tumours has attracted the interest of biomathematicians. Tumour resection has been investigated by Woodward et al. ${ }^{67}$ and Swanson et al. ${ }^{60}$ The works of Tracqui et $a l .{ }^{63}$ and Swanson et al. ${ }^{58}$ provide an extensive analysis of the chemotherapy of brain tumours. Anti-angiogenic therapies have been modelled and analysed by Arakelyan et al. ${ }^{5}$ Finally, some researchers modelled mathematically the pharmacokinetics of drug delivery to various tumour sites, e.g. Owen et al. ${ }^{42}$

\section{Mathematical Modelling: Glioblastoma Multiforme}

In recent years researchers have developed several types of GBM models. Mathematical modelling allows the treatment of diverse biological questions concerning the analysis of early GBM growth, therapy effectiveness or even simulations in realistic brain structure and geometry.

\subsection{Early glioma tumour growth}

This section attempts to collect and critically discuss studies that model the early growth of gliomas. The following models are mostly cellular automata ${ }^{a}$ or

\footnotetext{
${ }^{a}$ A cellular automaton is a discrete dynamical system. Space, time, and the states of the system are discrete. Each point in a regular spatial lattice, called a cell, can have any one of a finite number of states. The states of the cells in the lattice are updated according to a local rule. That is, the state of a cell at a given time depends only on its own state one time step previously, and the states of its nearby neighbors at the previous time step. All cells on the lattice are updated synchronously. Thus the state of the entire lattice advances in discrete time steps.
} 
agent-based $^{\mathrm{b}}$ models that capture the glioma growth evolution from the very beginning (first cell or a small set cells) of the disease. Cell-based modelling allows the researcher to identify the self-organizational behaviour of a cell system (tumour) geared by micro-dynamics (cellular or even molecular interactions).

(a) GBMs form complex growth patterns in vitro (multicellular spheroids) in which invasive cells organize in tenuous branches. Sander et $a$ l. $^{49}$ formulate a chemotaxis model (the evolution of the system is affected by the gradient of nutrient concentration) for this sort of growth. A key element for pattern generation is the homotypic attraction, i.e. the tendency of invasive cells to follow pathways previously explored. The authors examine the existence of an intrinsic instability in the model that leads to branch formation, similar to in vitro experiments.

The results of this paper show that both strong chemotaxis and strong homotypic attraction are required for branch formation within the invasive zone. Model simulations resemble the morphology of multicellular spheroid experiments, showing a DLA-like branching formation (DLA = Diffusion Limited Aggregation ${ }^{66}$ ). As the authors note, the homotypic attraction is not convincingly modelled. Thus, due to the roughness of the model, only qualitative results are obtained. However, this approach would allow to model various other phenomena related to GBM development, such as haptotaxis or tissue mechanical effects.

(b) The work of Kansal et al. $(2000)^{22}$ models the 3D evolution of gliomas by developing a cellular automaton. Initiating the system from a small number of cells, one can follow the evolution to a fully developed tumour ( $10^{11}$ cells). An appropriate space discretization leads to a flexible, adaptive lattice, able to simulate realistic geometries. The model key outputs are: average tumour radius, proliferating rim thickness and necrotic fraction. For the calculation of the model outputs, the simulation utilizes four microscopic parameters: cell division probability (linked to the cell-division times), necrotic parameter (controlled by nutrient needs), proliferative parameter (controlled by nutrient needs) and maximum tumour extent (controlled by pressure response). The model distinguishes necrotic and non-tumourous cells, proliferative and non-proliferative tumourous cells. Within the tumour three homocentric circular regions are identified: necrotic, proliferative and an outer non-proliferative respectively. The radius of each region is influenced by the values of the corresponding parameters. This work was the first 3D cellular automaton model of solid glioma tumour growth, which realistically models the macroscopic behaviour of a malignant tumour using predominantly microscopic parameters. The parameters of the cellular automaton transition rules are fitted from experiments.

\footnotetext{
${ }^{\mathrm{b}}$ In agent-based modelling, agents are computational units (e.g. cells) that decide for themselves under a set of rules. The collective behaviour of the agents defines the macroscopic patterns of the whole system (e.g. tumour). Like CAs, the system evolution can be realised on a lattice.
} 
However, the transition rules are nonlocal, i.e. they are not defined by neighbouring cell interactions but depending on the structure of the whole tumour. A crucial disadvantage of this model is its inability to properly describe invasion by a single cell. Note that, after glioma resection, some isolated invasive cells are left behind and will eventually cause recurrence of the tumour.

(c) Agent-based modelling is used by Mansury et $a l^{30}$ in order to model and realistically simulate early avascular GBM growth. The introduction of variable lattice node distances enables simulations on realistic brain geometries, by inhibiting a generic flexibility to the model. A fitness function defines the attractivity of each node, by considering nutrient and toxic concentrations and mechanical confinements at each lattice node, and affects the process of cell movement (the agents-cells are looking for nodes with higher fitness scores). The authors introduce two kinds of search mechanisms in order to simulate local and global interactions between the tumour cells. Moreover, the model includes microscopic processes such as genetic mutations (of cell phenotypes) that lead to new malignant cell sub-populations. Interestingly, the authors numerically explore the parameter space and they locate a phase transition (tumour cell condensation) at critical nutrient and toxic concentrations.

The simulations provide insight into the connection between fractal surface dimensions and average invasion speed. Due to the multi-scaled nature of the model, the authors can show that it is the invasive and not the proliferative cell phenotype that drives the tumour to spatial expansion, as indicated by strong correlations between tumour diameter and gene expression profiles of migrating tumour cells. Although the model is very complex, the simulations do not yet agree quantitavely with clinical data.

\subsection{Invasion}

Invasion of brain parenchyma by tumour cells is one of the most dominant aspects of GBM growth. The clinical aggressiveness of this brain tumour prioritises the modelling of invasion dynamics. A theoretical framework of invasion was introduced first by Tracqui et al. ${ }^{63}$ Woodward et al. ${ }^{67}$ Burgess et al. ${ }^{9}$ The early GBM models mentioned so far deal with invasion into a homogeneous brain structure. Recently, researches have also been concentrated on invasive tumour patterns in more complex brain structures.

(a) The work of Swanson et al. ${ }^{57}$ sheds light on glioma invasion into a nonhomogeneous brain structure. Using the BrainWeb brain atlas, the authors simulate a realistic brain geometry including fibre differentiation into grey and white matter (inhomogeneous structure). The main assumption of this model is that tumour cells move with different speeds in grey and white matter. The model is a one-component (tumour cells) reaction-diffusion equation that describes cell proliferation and space-dependent diffusion. The invasive behaviour is approximated by the diffusion term and the proliferation is characterised by 
the reaction term. Model analysis depicts waves of the tumour visible through MRI - by means of monitoring diffusion and proliferation rates. The authors compare macroscopic simulation data (volumes, survival time) with clinical data obtained from analysis of a series of one patient's CT scans. All the parameters are fitted to this specific data set. Thus, a general definition (independent of patient) of these parameters is not acquired. A comparison of the numerically calculated time-space malignant cell distribution with the corresponding distributions of the clinical tumour cases has not been carried out so far.

(b) A different approach was taken by Wurzel et al. ${ }^{68}$ This model aims to investigate the effect of brain structure inhomogeneity, as defined before. A CA model is developed simulating the invasion, proliferation and death of tumour cells. The introduction of a prepattern (orientation gradient field) models the heterogeneity of brain parenchyma and eventually leads to space-dependent glioma growth. The authors develop and analyse different scenarios of prepattern influence. The introduction of the prepattern increases the speed of the invading tumour front. For high fluxes of invading cells the model predicts the formation of cancer islets at distance from the main tumour bulk. This invasion pattern closely resembles the clinical situation. So far, no simulations have been conducted on realistic geometries.

\subsection{Tumour modelling of genetic alterations and their macroscopic effects}

An important theoretical and clinical challenge is the development of models that predict which microscopic (genetic) modifications are required for a given macroscopic behaviour. An interesting approach is using an event-guided evolution (the term "event" refers to a sequence of cell mutations) due to alterations of the genetic profile of the cells. Painter et al. ${ }^{44}$ developed a macroscopic model that describes several tumour-relevant quantities: malignant cell densities (of all grades), vascular density, necrotic tissue and chemical (nutrient and toxic) concentrations. Starting from grade I tumour cells, a stochastic sequence of genetic mutations can trigger a variety of scenarios that may even lead to the creation of GBM. In this framework, the authors attempt to analyse the effect of these genetic alterations at the macroscopic level, namely in terms of tumour cell concentration. Moreover, the authors propose a way of tumour grade diagnosis by histological analysis of tumour parts.

The appeal of this work lies in its multi-scaled nature. The authors develop a complete macroscopic mathematical description incorporating a stochastic sequence of genetic alterations (emerging increase in concentrations of malignant populations), in an attempt to "guess" which of these genetic mutations are relevant for leading us to the clinically observed phenotype. However, the authors do not attempt to find minimal classes of events that lead to certain scenarios, i.e. identify minimal genetic profiles that result in a certain tumour (more speciffically malignancy grade). 


\subsection{Modelling therapies}

In this section, we discuss the mathematical modelling of glioma chemotherapy, tumour resection and radiation therapy. Note, that glioma radiotherapy has not been modelled so far.

\subsubsection{Chemotherapy}

Many GBM patients are treated with chemotherapy at some point during the course of their disease. Often a combination of drugs is administered to the patient in order to reduce the emergence of tumour cell clones resistant to a specific cytostatic agent.

(a) Tracqui et al. ${ }^{63}$ have analysed a patient with recurrent anaplastic astrocytoma, who was treated by chemotherapy. Serial CT scans were obtained during the 12 terminal months of a patient, as he was receiving two different chemotherapies. The authors modelled chemotherapy as a spatially homogeneous (no assumption of differentiation into grey and white) equation of a reaction-diffusion system, namely: the rate of change of tumour cell population at a certain brain area (infinitesimally small) equals diffusion (motility) of tumour cells plus the net proliferation of tumour cells and loss of tumour cells due to chemotherapy. The serial CT scans determined the value of the parameters that best fit the model to the experimental data. The authors extended the model by the introduction of a second tumour cell population, sensitive to one chemotherapy but not the others. The model simulations agreed with the clinical data derived from CT scans. This work constitutes the first modelling attempt of glioma chemotherapy.

However, this approach also has several disadvantages. The parameter values refer to a specific patient and have been used as the probable average of all high-grade gliomas. Consequently, they can only be indicative for other cases. Extrapolating from parameters specific to one individual to more general ones is not a trivial task. A second drawback of the model is its treatment of the brain structure. Assuming homogeneous brain does not allow for realistic simulations. There is no explicit modelling of nutrient quantities or any kind of cell interactions (amongst tumour cells or extracellular matrix). Tumour cell motility is described as a random phenomenon (diffusion).

(b) Recently, Swanson et al. ${ }^{58}$ developed a model for chemotherapy of GBM tumours. The model incorporates all the properties of the Tracqui model plus the assumption of a heterogeneous brain structure. Grey and white brain matter differentiation makes the diffusion process space-dependent. Simulations were performed for realistic brain geometries, allowing for a better understanding of the therapy effect. Indeed, the authors could show that in certain situations drug delivery may be sufficient to result in a net decay of tumour cells in grey matter while still allowing for a net proliferation of the tumour cells in the white matter. 
Simulation results demonstrate that although the total number of tumour cells within the brain may be decreasing, the extent of invasion of the tumour cells remains practically unaffected due to the increased motility of those cells within the white matter regions. Like Tracqui and co-workers, also Swanson et al. use a set of serial CT scans from a specific patient to estimate the central parameters of the model. As discussed above this may limit any generalizations.

\subsubsection{Tumour resection effects}

Several groups have attempted to model the effects of various types of tumor resection. Note that invasive glial neoplasms cannot truly be completely resected; a "gross total resection" denotes that while all visible solid tumour has been removed, the invisible infiltrating tumour component has been left behind. "Subtotal" or partial resection implies that some of the grossly visible tumour has not been resected, and "biopsy" that practically no relevant amount of tumour tissue has been removed.

(a) The most prominent study on resection modelling was published by Woodward et $a l .{ }^{67}$ The model uses the methodology of Tracqui et $a l .{ }^{63}$ (reaction-diffusion equation), except that it simulates only one kind of malignant cell population. The parameter estimation is based on the same patient's CT scans of Tracqui et al. ${ }^{63}$ with the hypothesis that $\pm 50 \%$ variations in diffusion and proliferation rates would encompass essentially all of the patients with high-grade gliomas.

The authors investigate several scenarios of GBM resection and simulate tumour recurrence. The model suffers from the same drawbacks as the study by Traqui et al. ${ }^{63}$ : the CT data from one specific patient do not prove the universal applicability of the model; it may be able to describe the clinical course of a specific patient case but a generalization is certainly not trivial. The model also assumes a homogeneous brain structure, i.e. no fibre differentiation. In spite of these objections, the shapes of the curves of survival of groups of patients and their medians were captured by the model.

(b) The natural extension of the Woodward et al. ${ }^{67}$ study was the introduction of an inhomogeneous brain structure. Swanson et al. ${ }^{60}$ enriched the above-mentioned approach by the distinction of white and grey brain matter that makes the diffusion process dependent on brain structure. Numerical analysis of the model, with different diffusion and growth rates of the malignant population, show consistent recurrence of the resected tumour (after both gross total and extensive subtotal resections). The model allows to predict the expected locations of emerging malignant cell populations and their corresponding densities. The definition of the parameters is based on the same methodology that was used in the previous studies of Swanson et al. . $^{57,58,60,62}$ 


\section{Discussion}

Mathematical modelling of gliomas aims at a better and more profound understanding of the underlying biological processes. The knowledge derived from such models may prove essential for biologists and physicians alike to develop innovative treatments against this disease. Although a technical review of the implemented mathematical methods may lead to fruitful discussions between biomathematicians, it may overlook the main target group for this kind of research, the biomedical researchers. Hence, we considered it imperative to review the present GBM modelling research in a way that is useful for both theoreticians and practitioners.

Our classification takes into account the main medical and biological focus of each study, rather than the mathematical approach chosen. We distinguish between the following classes/categories:

- Early tumour growth: The literature contains several studies that model and simulate early GBM growth. These studies explain the growth patterns seen in experiments and effectively simulate the avascular early phase of glioma growth. Although cell-based modelling usually delivers convincing qualitative simulations, considerable efforts will be required to achieve exact quantitative results (e.g. Kansal et $\left.a ._{.}^{22}\right)$.

- Tumour invasion: Invasion has been modelled both at the cellular and at the tissue level. The effect of the inhomogeneous structure of the brain (e.g. grey and white matter differentiation) on glioma invasion, has been studied extensively. The brain topology dictates diffusion rates, accelerating the invasion into white matter. The structure of the brain can also be modelled as an orientation gradient field parallel to the white matter fiber tracks, which facilitates invasion/migration of the tumour cells (e.g. Wurzel et al. ${ }^{68}$ ). These approaches provide a concrete framework for a better understanding of tumour invasion and may have implications for the design of future therapies.

- Genetic mutations and their macroscopic effects: In this category, the goal is to model a series of genetic mutations and simulate the resulting macroscopic GBM phenotype. Painter et $a l .{ }^{44}$ investigated this problem and described various scenarios starting with a specific sequence of genetic mutation that ultimately lead to certain tumour grades and growth patterns. The next step would be the formulation of minimal event classes (i.e. gene mutations) that reflect specific tumour behaviours.

- Therapy: Modelling efforts are invaluable in testing and designing treatment strategies theoretically, i.e. before they are implemented clinically. GBM chemotherapy and tumour resection, have been analysed in theoretical studies. As far as modelling the effects of chemotherapy is concerned, the early approaches described glioma growth and invasion under the approximation of homogeneous brain tissue, with or without gross anatomical boundaries. Considering complex heterogeneous tissue, with varying proportions of grey and white matter, provided better insight into the effects of therapy. GBM resection has been investigated 
also under the assumption of a diffusive tumour in a homogeneous as well as in an inhomogeneous brain structure. These models (e.g. Swanson et al. ${ }^{60}$ ) correctly predict the site of a tumour recurrence by describing the location of malignant tumour cell populations and their densities. Quite in accordance with clinical experience, gliomas appear uncurable in these models by resection, no matter how extensive, due to their infiltrative nature.

The reader should realize that mathematical modelling offers significant insight into glioma evolution, but so far nobody can claim that it provides a full picture of the disease. In addition several crucial questions have not been adequately addressed so far by modelling efforts (and hence, have not been treated in this review up to this point):

- Glioma oncogenesis: Why do GBMs form? What are the mechanisms responsible? E.g. Koptioug et $a .^{24}$ interpret oncogenesis as a phase transition in a dynamical system.

- Evolution and complexity of the GBM phenotype: Why do gliomas appear to have such a great ability of self-organisation and re-organisation? What are the properties of the glial cell network that allow such behaviour?

- The stability of a GBM: Why are gliomas so robust (i.e. resistant) against perturbations (i.e. therapies)? Is it possible to design intelligent therapies (at the cellular level) that disturb the tumour's robustness?

These questions create a new agenda for glioma research.

Finally, the clinician needs accurate predictions. Tumour modelling culminating in highly complex and time-consuming simulations will probably not be the answer. Studies considering only the putatively essential ingredients of GBM growth, but validated with actual clinical data may be helpful in this regard. We sincerely hope that a more profound knowledge of such central tumour characteristics will eventually lead to the design of more effective therapeutic strategies.

\section{References}

1. J. A. Adam and N. Bellomo, A survey of models for tumor-immune system dynamics, in Introduction to Deterministic Tumor (or Spheroid) Growth Models (Birkhäuser, 1997).

2. T. Alarcon, H. M. Byrne and P. K. Maini, A multiple scale model for tumour growth, J. Theor. Biol. 225 (2003) 257-274.

3. D. Ambrosi, N. Bellomo and L. Preziosi, Modelling tumor progression, heterogeneity and immune competition, J. Theor. Med. 4 (2002) 51-65.

4. A. R. A. Anderson, Solid tumour invasion: The importance of cell adhesion, in Function and Regulation of Cellular Systems: Experiments and Models, eds. A. Deutsch, M. Falcke, J. Howard and W. Zimmermann (Birkhäuser, 2003).

5. L. Arakelyan, V. Vainstein and Z. Agur, A computer algorithm describing the process of vessel formation and maturation, and its use for predicting the effects of 
anti-angiogenic and anti-maturation therapy on vascular tumour growth, Angiogenesis 5 (2002) 203-214.

6. N. Bellomo, E. De Angelis and L. Preziosi, Multiscale modeling and mathematical problems related to tumour evolution and medical therapy, J. Theor. Med. 5 (2004) $111-136$.

7. BrainWeb, Simulated Brain Database, http://www.bic.mni.mcgill.ca/brainweb/.

8. A. Bru, S. Albertos, J. L. Subiza, J. L. Garcia-Asenjo and I. Bru, The universal dynamics of tumour growth, Biophys. J. 85 (2003) 2948-61.

9. P. K. Burgess, P. M. Kulesa, J. D. Murray and E. C. Alvord, The interaction of growth rates and diffusion coefficients in a three-dimensional mathematical model of gliomas, J. Neuropathol. Exp. Neurol. 56 (1997) 704-713.

10. H. M. Byrne and M. A. J. Chaplain, Mathematical models for tumour angiogenesis: numerical simulations and nonlinear wave solutions, Bull. Math. Biol. 57 (1995) 461-486.

11. M. S. Corcoran, M. D. Rolando and F. Del Maestro, Testing the 'GO OR GROW' hypothesis in human medulloblastoma cell lines in two and three dimensions, Neurosurgery 53 (2003) 174-184.

12. T. Delmuth, N. J. Hopf, O. Kempski, D. Sauner, M. Herr, A. Giese and A. Perneczky, Migratory activity of human glioma cell lines in vitro assessed by continuous single cell observation, Clin. Exper. Metastas. 18 (2001) 589-597.

13. A. Deutsch and S. Dormann, Cellular Automaton Modeling of Biological Pattern Formation (Birkhäuser, 2004).

14. S. Dormann and A. Deutsch, Modeling of self-organized avascular tumor growth with a hybrid cellular automaton, In Silico Biol. 2 (2002) 0035.

15. D. Drasdo, S. Hhme, S. Dormann and A. Deutsch, Cell-based models of avascular tumor growth, in Function and Regulation of Cellular Systems: Experiments and Models, A. Deutsch, M. Falcke, J. Howard, and W. Zimmermann eds. (Birkhauser, 2003).

16. J. Folkman and M. Hochberg, Self-regulation of growth in three dimensions, J. Exp. Med. 138 (1973) 745-753.

17. A. Gamba, D. Ambrosi, A. Coniglio, A. de Candia, S. di Talia, E. Giraudo, G. Serini, L. Preziosi and F. Bussolino, Percolation, morphogenesis, and Burgers dynamics in blood vessel formation, Phys. Rev. Lett. 90 (2003) 118101.

18. A. Giese, L. Kluwe, B. Laube and M. H. Berens, Migration of human glioma cells on myelin, Neurosurg. 38 (1996) 755-764.

19. A. Giese, M. A. Loo, N. Tran, D. Haskett and S. W. Coons, Dichotomy of astrocytoma migration and proliferation, Int. J. Cancer 67 (1996) 275-282.

20. A. Giese and M. Westphal, Glioma invasion in the central nervous system, Neurosurgery 39 (1996) 235-50.

21. A. R. Kansal, S. Torquato, E. A. Chiocca and T. S. Deisboeck, Emergence of a subpopulation in a computational model of tumor growth, J. Theor. Biol. 207 (2000) 431-441.

22. A. R. Kansal, S. Torquato, G. R. Harsh IV, E. A. Chiocca and T. S. Deisboeck, Simulated brain tumor growth dynamics using a three-dimensional cellular automaton, J. Theor. Biol. 203 (2000) 367-382.

23. P. Kleihues and W. K. Cavenee, World Health Organization classification of tumours, pathology and genetics, in Tumours of the Nervous System (IARC Press, 2004).

24. A. V. Koptioug, E. Mamontov, Z. Taib and M. Willander, The phase-transition morphogenic model for oncogeny as a genotoxic homeostatic dysfunction: Interdependence of modeling, advanced measurements, and numerical simulation, in ICSB2004, 5th Int. Conf. Systems Biology, 9-13 October 2004, Heidelberg, Germany. 
25. M. Lacroix et al., A multivariate analysis of 416 patients with glioblastoma multiforme: prognosis, extent of resection, and survival, J. Neurosurg. 95 (2001) 190-8.

26. E. Mandonnet, J. Y. Delattre, M. L. Tanguy, K. R. Swanson, A. F. Carpentier, H. Duffau, P. Cornu, R. Van Effenterre, E. C. Alvord, Jr. and L. Capelle, Continuous growth of mean tumor diameter in a subset of WHO grade II gliomas, Ann. Neurol. 53 (2003) 524-528.

27. D. Manoussaki, A mechanochemical model of vasculogenesis and angiogenesis, Math. Model. Num. Anal. 37 (2003) 581-599.

28. Y. Mansury, M. Kimura, J. Lobo and T. S. Deisboeck, Emerging pattern in tumor systems: Simulating the complex dynamics of multicellular cluster structures by using a novel agent-based spatio-temporal agglomeration model, J. Theor. Biol. 219 (2002) 343-370.

29. Y. Mansury and T. S. Deisboeck, The impact of 'search precision' in an agent-based tumor model, J. Theor. Biol. 224 (2003) 325-337.

30. Y. Mansury and T. S. Deisboeck, Modelling tumours as complex biosystems: An agent-based approach, in Complex Systems Science: The Emergence of a New Approach in BioMedicine, eds. J. Y. Kresh and T. S. Deisboeck (Kluwer, 2004).

31. B. P. Marchant, J. Norbury and A. J. Perumpanani, Traveling shock waves arising in a model of malignant invasion, SIAM. J. Appl. Math. 60 (2000) 263-276.

32. B. P. Marchant, J. Norbury and J. A. Sherratt, Travelling wave solutions to a haptotaxis-dominated model of malignant invasion, Nonlinearity 14 (2001) $1653-1671$.

33. M. Marusic, Z. Bajzer, J. P. Freyer and S. Vuk-Pavlovic, Analysis of growth of multicellular tumour spheroids by mathematical models, Cell Prolif. 27 (1994) 73-94.

34. Y. Matsukado, C. S. MacCarty and J. W. Kemohan, The growth of glioblastoma multiforme (astrocytomas, grades 3 and 4) in neurosurgical practice, J. Neurosurg. 18 (1961) 636-644.

35. E. Medico, A. Gentile, C. Lo Celso, T. A. Williams, G. Gambarotta, L. Trusolino and P. M. Comoglio, Osteopontin is an autocrine mediator of hepatocyte growth factor-induced invasive growth, Cancer Res. 61 (2003) 5861-8.

36. T. Mikkelsen, S. A. Enam and M. L. Rosenblum, Invasion in malignant glioma, in Youman's Neurological Surgery, ed. H. R. Winn (W. B. Saunders Co., 2003).

37. J. Moreira and A. Deutsch, Cellular automaton models of tumor development: A critical review, Adv. Compl. Syst. 5 (2002) 247-267.

38. MRC Brain Tumour Working Party, Randomized trial of procarbazine, lomustine, and vincristine in the adjuvant treatment of high-grade astrocytoma: A Medical Research Council trial, J. Clin. Oncol. 15 (2001) 509-18.

39. J. D. Murray, Mathematical Biology, 3rd edn. (Springer-Verlag, 2002).

40. M. E. Orme and M. A. J. Chaplain, A mathematical model of the first steps of tumour-related angiogenesis: Capillary sprout formation and secondary branching, IMA J. Math. App. Med. Biol. 13 (1996) 73-98.

41. M. E. Orme and M. A. J. Chaplain, Two-dimensional models of tumour angiogenesis and anti-angiogenesis strategies, IMA J. Math. App. Med. Biol. 14 (1997) 189-205.

42. M. R. Owen, H. M. Byrne and C. E. Lewis, Mathematical modelling of the use of macrophages as vehicles for drug-delivery to hypoxic tumour sites, J. Theor. Biol. 226 (2004) 377-391.

43. A. S. Qi, X. Zheng, C. U. Du and B. S. An, A cellular automaton model of cancerous growth, J. Theor. Biol. 161 (1993) 1-12.

44. K. J. Painter, N. Savill and E. Shochat, Computing evolutions in brain tumours, submitted article (2005). 
45. A. J. Perumpanani, J. A. Sherratt, J. Norbury and H. M. Byrne, Biological inferences from a mathematical model of malignant invasion, Invas. Metast. 16 (1996) 209-221.

46. A. J. Perumpanani, J. A. Sherratt, J. Norbury and H. M. Byrne, A two parameter family of travelling waves with a singular barrier arising from the modelling of extracellular matrix mediated cellular invasion, Phys. D 126 (1999) 145-159.

47. Ed. L. Preziozi, Cancer Modelling and Simulation (Chapman \& Hall/CRC, 2003).

48. D. S. Russell and L. J. Rubinstein, Pathology of Tumors of the Nervous System, 5th edn. (Williams \& Wilkins, 1989).

49. L. M. Sander and T. S. Deisboeck, Growth patterns of microscopic brain tumours, Phys. Rev. E66 (2002) 051901.

50. H. J. Scherer, Structural development in gliomas, Am. J. Cancer. 34 (1938) 333-351.

51. H. J. Scherer, The forms of growth in gliomas and their practical significance, Brain 63 (1940) $1-35$.

52. J. A. Sherratt and M. A. Nowak, Oncogenes, anti-oncogenes and the immune response to cancer: A mathematical model, Proc. Roy. Soc. Lond. B 248 (1992) 261-271.

53. J. A. Sherratt and M. A. J. Chaplain, A new mathematical model for avascular tumour growth, J. Math. Biol. 43 (2001) 291-312.

54. D. L. Silbergeld and M. R. Chicoine, Isolation and characterization of human malignant glioma cells from histologically normal brain, J. Neurosurg. 86 (1997) 525-531.

55. J. Smolle and H. Stettner, Computer simulation of tumour cell invasion by a stochastic growth model, J. Theor. Biol. 160 (1993) 63-72.

56. R. Stupp, W. P. Mason, M. J. van den Bent, M. Weller, B. Fisher, M. Taphoorn, A. A. Brandes, G. Cairncross, D. Lacombe and R. O. Mirimanoff, Radiotherapy plus concomitant and adjuvant temozolomide for glioblastoma, N. Engl. J. Med. 352 (2005) 987-996.

57. K. R. Swanson, E. C. Alvord, Jr. and J. D. Murray, A quantitative model for differential motility of gliomas in gray and white matter, Cell Prolif. 33 (2000) 317-330.

58. K. R. Swanson, E. C. Alvord, Jr. and J. D. Murray, Quantifying efficacy of chemotherapy of brain tumors (gliomas) with homogeneous and heterogeneous drug delivery, Acta Biotheor. 50 (2002) 223-237.

59. K. R. Swanson, E. C. Alvord, Jr. and J. D. Murray, Virtual brain tumors (gliomas) enhance the reality of medical imaging and highlights inadequacies of current therapy, Brit. J. Cancer 86 (2002) 14-18.

60. K. R. Swanson, E. C. Alvord, Jr. and J. D. Murray, Virtual resection of gliomas: Effects of location and extent of resection on recurrence, Math. Comp. Model. 37 (2003) 1177-1190.

61. K. R. Swanson, C. Bridge, J. D. Murray and E. C. Alvord, Jr. Virtual and real brain tumors: Using mathematical modeling to quantify glioma growth and invasion, $J$. Neurol. Sci. 216 (2003) 1-10.

62. K. R. Swanson, E. C. Alvord, Jr. and J. D. Murray, Dynamics of a model for brain tumors reveals a small window for therapeutic intervention, Disc. Cont. Dyn. Syst. B 4 (2004) 289-295.

63. P. Tracqui, G. C. Cruywagen, D. E. Woodward, G. T. Bartoo, J. D. Murray and E. C. Alvord, Jr. A mathematical model of glioma growth: The effect of chemotherapy on spatio-temporal growth, Cell Prolif. 28 (1995) 17-31.

64. S. Turner and J. A. Sherratt, Intercellular adhesion and cancer invasion: A discrete simulation using the extended Potts model, J. Theor. Biol. 216 (2002) 85-100.

65. Weller et al., Neuro-Oncology Working Group 01 trial of nimustine plus teniposide versus nimustine plus cytarabine chemotherapy in addition to involved-field radiotherapy in the first-line treatment of malignant glioma, J. Clin. Oncol. 21 (2003) 3276-84. 
66. T. A. Witten and L. M. Sander, Diffusion-limited aggregation, a kinetic critical phenomenon, Phys. Rev. Lett. 47 (1981) 1400-1403.

67. D. E. Woodward, J. Cook, P. Tracqui, G. C. Cruywagen, J. D. Murray and E. C. Alvord, Jr. A mathematical model of glioma growth: The effect of extent of surgical resection, Cell Prolif. 29 (1996) 269-88.

68. M. Wurzel, C. Schaller, M. Simon, A. Deutsch, Cancer cell invasion of normal brain tissue: Guided by Prepattern?, J. Theor. Med. (2005) to appear.

69. K. J. Zuelch, Brain Tumors, 3rd edn. (Springer, 1986). 